\title{
A comprehensive gene expression atlas of sex- and tissue-specificity in the malaria vector, Anopheles gambiae
}

\author{
Dean A Baker ${ }^{1 *}$, Tony Nolan², Bettina Fischer ${ }^{3}$, Alex Pinder ${ }^{2}$, Andrea Crisanti $^{2}$ and Steven Russell ${ }^{1,3}$
}

\begin{abstract}
Background: The mosquito, Anopheles gambiae, is the primary vector of human malaria, a disease responsible for millions of deaths each year. To improve strategies for controlling transmission of the causative parasite, Plasmodium falciparum, we require a thorough understanding of the developmental mechanisms, physiological processes and evolutionary pressures affecting life-history traits in the mosquito. Identifying genes expressed in particular tissues or involved in specific biological processes is an essential part of this process.

Results: In this study, we present transcription profiles for $\sim 82 \%$ of annotated Anopheles genes in dissected adult male and female tissues. The sensitivity afforded by examining dissected tissues found gene activity in an additional $20 \%$ of the genome that is undetected when using whole-animal samples. The somatic and reproductive tissues we examined each displayed patterns of sexually dimorphic and tissue-specific expression. By comparing expression profiles with Drosophila melanogaster we also assessed which genes are well conserved within the Diptera versus those that are more recently evolved.

Conclusions: Our expression atlas and associated publicly available database, the MozAtlas (http://www.tissue-atlas. org), provides information on the relative strength and specificity of gene expression in several somatic and reproductive tissues, isolated from a single strain grown under uniform conditions. The data will serve as a reference for other mosquito researchers by providing a simple method for identifying where genes are expressed in the adult, however, in addition our resource will also provide insights into the evolutionary diversity associated with gene expression levels among species.
\end{abstract}

\section{Background}

For organisms in which large-scale mutagenic studies are problematic, gene expression catalogues are an important tool for annotating processes on a gene-bygene basis. In the malarial vector Anopheles gambiae, studies have focused on differential expression in males and females [1,2], on samples collected before and after the bloodmeal $[2,3]$ and in dissected tissues such as the midgut [2], salivary glands $[4,5]$, ovaries $[2,6]$, head and carcass $[7,8]$. However, since these studies often involve different mosquito strains, different experimental platforms and analysis by different statistical methods, comparison among treatments is challenging. Here, we

\footnotetext{
* Correspondence: dab55@cam.ac.uk

'Department of Genetics, University of Cambridge, Downing Street, Cambridge CB1 3QA, UK

Full list of author information is available at the end of the article
}

provide a comprehensive expression atlas and associated publicly available database, the MozAtlas (http://www. tissue-atlas.org), cataloguing the relative strength and specificity of gene expression in tissues of male and female mosquitoes using a single genome-wide platform, protocol and analysis.

We employed transcriptional profiling to analyse RNA levels in whole body mosquito samples, eight separate somatic tissues (head, salivary gland, midgut, Malpighian tubules, thoracic and abdominal carcass) and the reproductive tissues (testis, accessory gland, ovary) of males and females separately. In common with the majority of sexually reproducing organisms, Anopheles has specialized reproductive traits. Of particular interest is the female-specific activity of blood-feeding, which provides protein for egg development and is a key determinant in Plasmodium transmission. In contrast, male mosquitoes
C Biomed Central

() 2011 Baker et al; licensee BioMed Central Ltd. This is an Open Access article distributed under the terms of the Creative Commons Attribution License (http://creativecommons.org/licenses/by/2.0), which permits unrestricted use, distribution, and reproduction in any medium, provided the original work is properly cited. 
feed entirely on sugar, are not adapted for digesting blood and do not transmit malaria. Consequently, those tissues involved in acquiring, ingesting and digesting blood are expected to display substantial sexual dimorphism at the level of gene expression.

In this paper we summarize the functions and sequence level divergence of genes with sexually dimorphic or tissue enriched expression patterns to determine which genes, if any, are rapidly evolving. In addition, by comparing Anopheles expression profiles with matched tissues in Drosophila melanogaster, we assess evolutionary conservation of expression profiles within the Diptera and identify genes recently evolved in Anopheles with tissue specific patterns of expression. Such traits provide ideal candidates for use in population control, where vital or fertility-related genes may be targeted by genetic knockout [9]. With the ongoing development of insect genetics it has become increasingly likely that some pest populations, including mosquitoes, may be controlled with genetic modification [10-15].

\section{Results}

\section{Gene expression coverage}

We have analysed gene expression among Anopheles males and females using Affymetrix whole-genome microarrays. The microarray platform contains 16,942 unique Anopheles probes corresponding to 10,622 of the annotated protein-coding genes, equating to $82 \%$ of the genes in the genome. Female tissues were dissected at 24 hour intervals for a three day period following the blood-meal to provide information on the relative strength and specificity of gene expression in adult mosquito tissues throughout oogenesis. Equivalent male tissues were dissected from siblings in parallel. Array quality was first assessed by calculating the Pearson correlation coefficient between samples. Gene expression was highly similar among replicates $(R>=0.92)$, indicating that variation in our experiment was low (Additional File 1). While indicating biological replicates are highly consistent, individual-to-individual variation in gene expression will of course be masked by the effect of tissue pooling. After quality control, we detected expression of 10,031 probes corresponding to 7253 unique genes across Anopheles tissues. Hierarchical clustering with probe intensities indicates good discrimination of tissues, with expression distributed according to tissue and sex (Additional File 2). The fraction of expressed genes varied from $51 \%$ to $74 \%$ among samples (Figure 1B). Corresponding Drosophila organs analyzed on a similar array platform using the same normalization procedure, found similar levels of relative gene activity. Approximately 20\% of Anopheles transcripts in dissected samples are absent from whole-body
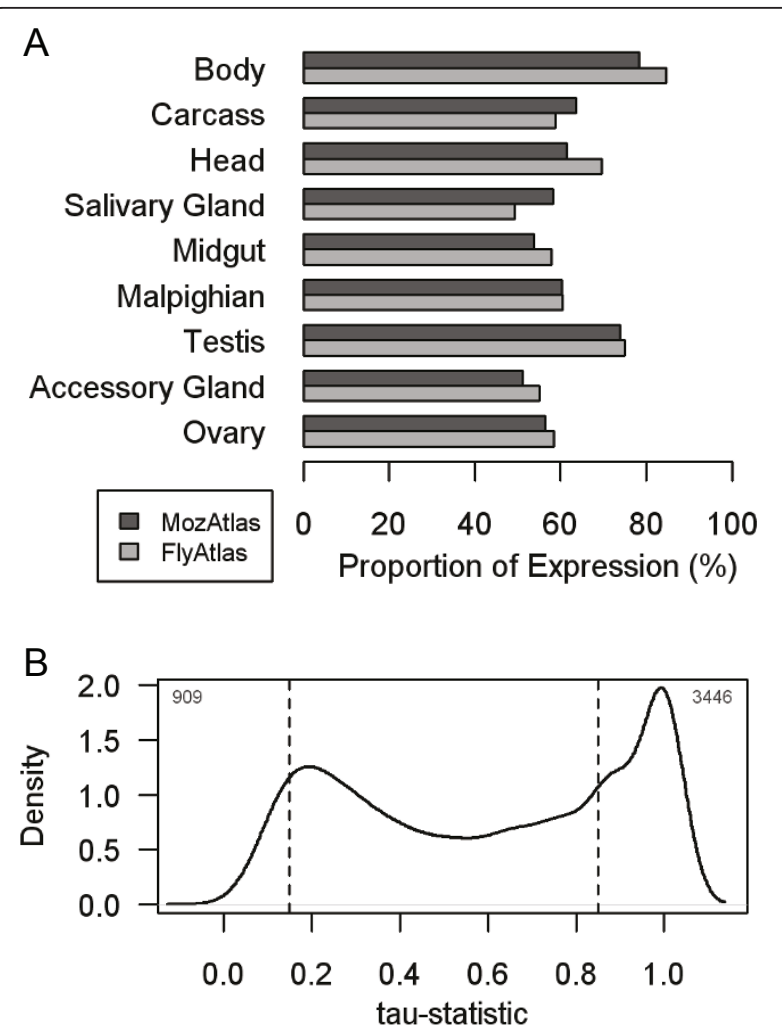

Figure $1 \mathrm{Global}$ expression coverage. (A) The proportion of probes giving at least 3 out of 4 mismatch calls in either male or female samples for tissue in the MozAtlas and FlyAtlas. (B) Tissue breadth. "House-keeping" genes were identified to have a taustatistic under 0.15 ( $n=909)$, and narrow expression a tau-statistic above $0.85(n=3446)$. Overall, only a third of genes were detected in all tissues.

estimates, and only a third of transcripts are recorded across all tissues (Figure 1B).

\section{Sexually dimorphic gene expression}

To investigate sexually dimorphic expression, a linear model was fit to male and female tissue samples. On the basis of differential expression, we identified probes as either male-biased or female-biased with a 2-fold change of intensity and statistical significance at the $\mathrm{Q}<0.05$ level (Additional File 3). Overall, 54\% of genes are sexually dimorphic in at least one organ, including a substantial degree of sex-biased expression in most somatic tissues. Of the 3924 sexually dimorphic genes, $72 \%$ are detected in whole-body male and female samples, with the remaining $28 \%$ only in dissected tissues (Figure 2A). Each tissue displays a moderate degree of sexual dimorphism, however, by and large, somatic tissues are closely related irrespective of sex when clustered according to expression level (Figure 2B). Thus, each tissue exhibits a specific gene expression profile that is overlaid with sex-specific functions. Sexually dimorphic expression is most skewed 


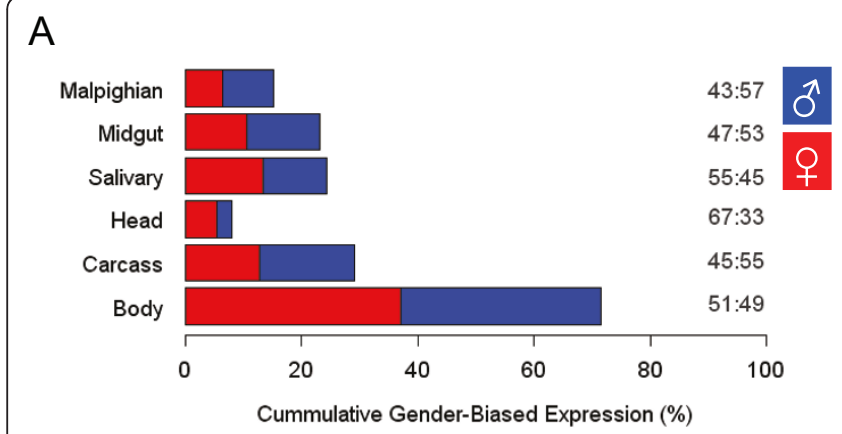

C

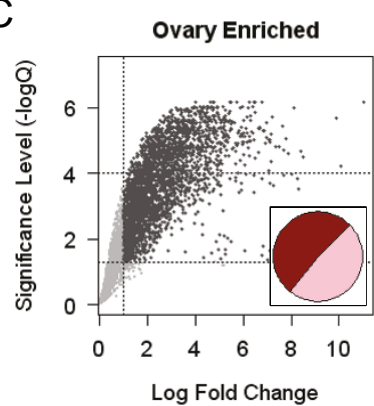

B

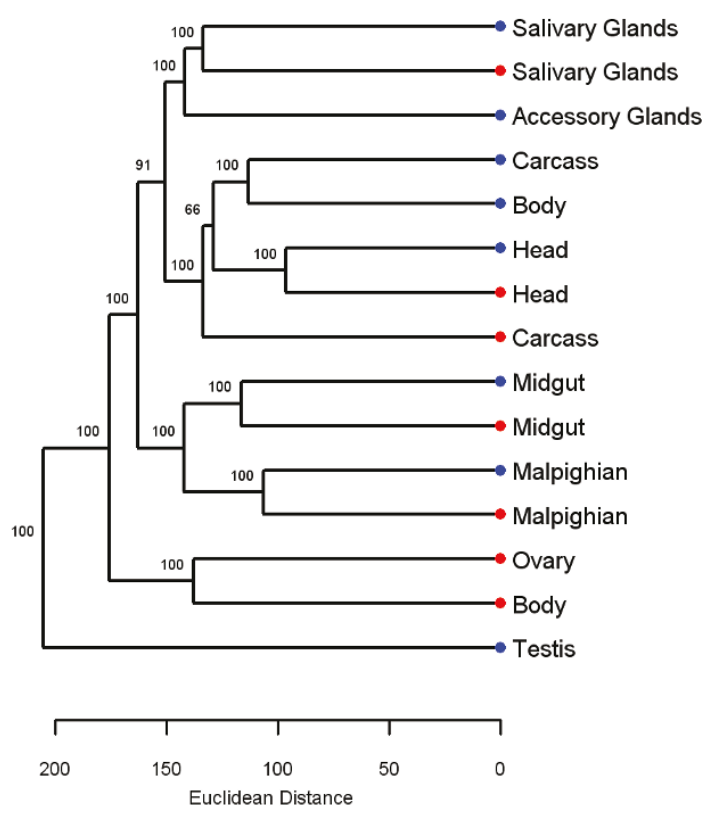

Figure 2 Sexually dimorphic expression. (A) The proportion of sexually dimorphic expression in each tissue versus total sexual dimorphism. The ratio of female- to male-biased expression is provided (female:male). All ratios deviate significantly from equality (Chi-squared test; $\mathrm{P}<0.05)$. (B) Hierarchical clustering of probes among tissues and sex with Euclidean Distance; female (red), male (blue). Branch support was estimated with 10,000 bootstrapped replicates. Expression enrichment against carcass for the (C) ovary and (D) testis. Significant gonad enrichment is highlighted in dark grey (ANOVA; $M>2 ; \mathrm{Q}<0.05$ ). Inset figures show the proportion of gonad enrichment which is also sexually dimorphic in whole-body samples, i.e. 51\% female-biased (dark red); $7 \%$ male-biased expression (dark blue).

in the head, with a high number of female-biased genes detected; in particular we found an over-representation of odorant receptor genes with female biased expression. However, overall, and in all other somatic tissues, there was approximately equal numbers of male- and femalebiased genes.

Sexual dimorphism at the gene expression level is associated with different and distinct functional categories (Additional File 4). For example, genes with 'digestion', 'protein metabolism' and 'proteolytic' functions, especially 'serine-type endopeptidase' are over-expressed in the midgut of females. Genes enriched for 'cellular homeostasis', 'ligase activity' and 'transporter activity' are enriched in the female salivary gland, while the malpighian tubules display an over-representation of genes associated with 'ion transportation'. In comparison, maleelevated genes are largely associated with 'carbohydrate metabolic activity', 'ion transporter activity' and 'iron ion binding' within the midgut, salivary gland and Malpighian tubules, as well as the carcass. Ultimately, many of the genes elevated in either sex are of unknown function.

\section{Tissue specific gene expression}

In the somatic and reproductive organs examined, a subset of genes showed considerable specificity (Figure
3A). The highest proportion of tissue-specific expression occurs in the testis, where approximately $10 \%$ of transcripts are unique. In comparison, ovary specific genes account for $\sim 4 \%$ of expression in the tissue, and several of the ovary-specific genes are members of the chorion family. We also found a set of 54 accessory-gland expressed genes, absent from other tissues, representing $\sim 2 \%$ of the expression in this tissue (Figure 3A). In common with the Drosophila Acps, our Anopheles candidates are over-represented in the top $10 \%$ of intensity values recorded for the accessory gland $\left(\chi^{2}=9.45\right.$; d.f. $=1$; $\mathrm{P}<$ $0.003)$, and many contain secretory domains necessary for transfer to females. Non-reproductive tissues also have a substantial number of genes with specific expression patterns, the majority in a single sex: these are especially prevalent in the midgut, salivary gland and carcass.

Previous studies indicate that genes with restricted expression have elevated rates of sequence divergence amongst related species [16]. We conducted a largescale survey of SNP A/S ratios using data from dbSNP to determine if such genes were evolving rapidly in Anopheles [17]. First, 11,224 genes with at least one coding SNP were collected. In total, $\sim 100,000$ coding-region SNPs and 316,043 intronic SNPs were identified, corresponding to SNP densities of 5.6 and 7.19 SNPs, 


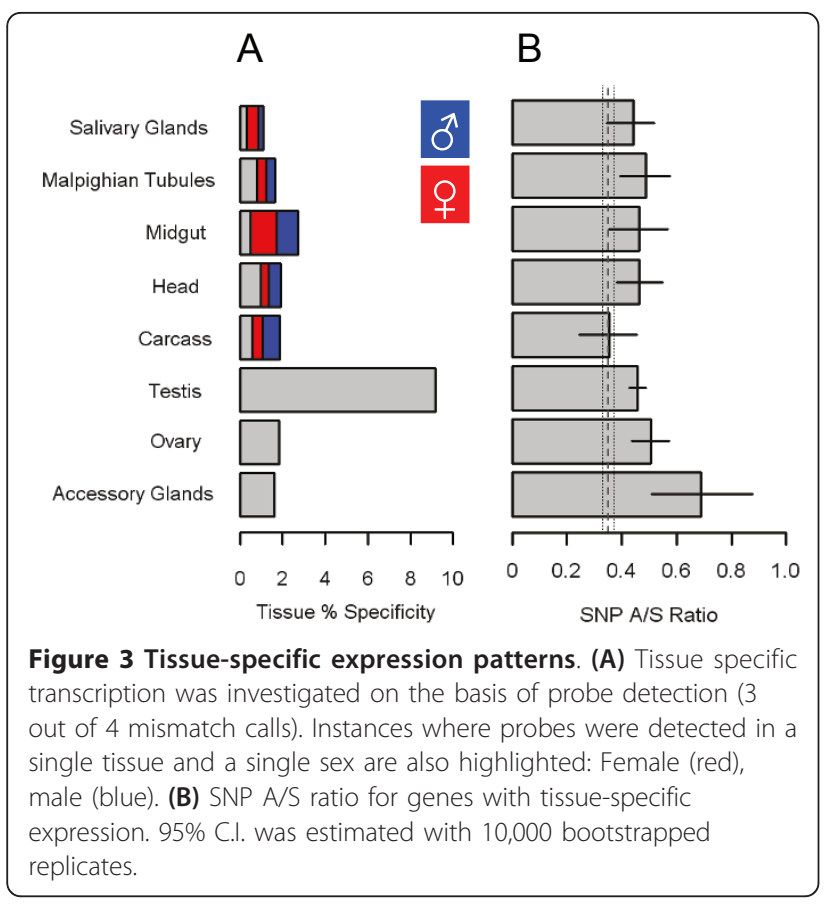

respectively, per 1,000 nucleotides. For our entire dataset, the number of non-synonymous coding SNPs per non-synonymous site (A) was 0.0033 , the number of synonymous coding SNPs per synonymous site (S) was 0.0068 , and the A/S ratio was 0.49 .

SNP A/S estimates of $<1$ suggest that most nucleotide substitutions have been eliminated by selection, i.e. purifying selection, whereas SNP A/S > 1 indicate that non-synonymous nucleotide substitutions have been maintained, i.e. positive selection. As expected, many tissue-specific genes display a higher ratio of A/S SNP ratio than those ubiquitously expressed throughout the organism, i.e. fewer non-synonymous mutations have been eliminated by selection and are evolving more rapidly (Figure 3B). For example, genes expressed in reproductive tissues including the testis, ovary and the male accessory gland have the highest rates of sequence divergence within Anopheles. Non-reproductive tissues including the head and Malpighian tubules show less deviation, while genes specifically expressed in the salivary gland and midgut have only marginally higher $\mathrm{A} / \mathrm{S}$ SNP ratios than ubiquitously expressed genes.

\section{Chromosomal distribution of tissue expression}

Across a range of Metazoan species, genes with elevated male expression are non-randomly distributed around the genome [18]. However, in Anopheles, previous global estimates of sex-biased expression failed to identify this property [1]. Anopheles tissue dissections provide substantially more information about male-specific gene expression than whole-body samples. For example, while a comparison of ovary and carcass expression indicates over half the ovary-enriched genes are female-biased in whole-body samples (Figure 2C), less than 10\% of testisenriched genes are male-biased, largely because they are undetected in whole-body samples (Figure 2D). Our new dataset allowed us to revisit the issue of genome position and expression in reproductive and somatic tissues. We found that genes expressed in the testis, but not the ovary, are under-represented on the $X$ chromosome (Figure 4A). In addition, male-biased somaticallyexpressed genes are also under-represented on the $X$ chromosome (Figure 4B). We find that SNP polymorphisms in testis-expressed genes show higher $\mathrm{A} / \mathrm{S}$ ratios on the $X$ chromosome than on the autosomes $\left(\chi^{2}=26.5 \mathrm{df}\right.$ $=1, \mathrm{P}<2.54 \times 10^{-7}$; Figure $\left.4 \mathrm{C}\right)$. Even though this finding is consistent with the expectation that $X$ chromosomes are hostile to testis-expressed genes, the same pattern was not observed with somatic tissues $\left(\chi^{2}=0.13\right.$ $\mathrm{df}=1, \mathrm{P}=\mathrm{NS}$; Figure 4D).

\section{Comparative evolution with Drosophila melanogaster}

To estimate evolutionary divergence in tissue expression profiles, orthology relationships in Drosophila and Anopheles (Insecta: Diptera) were traced back to a common Metazoan ancestor; Tribolium casteneum (Insecta: Coleoptera), Apis melifera (Insecta: Hymenoptera) or Caenorhabditis elegans (Nematoda: Rhabditida) (Figure 5A). From this analysis, we estimate that over half of the genes

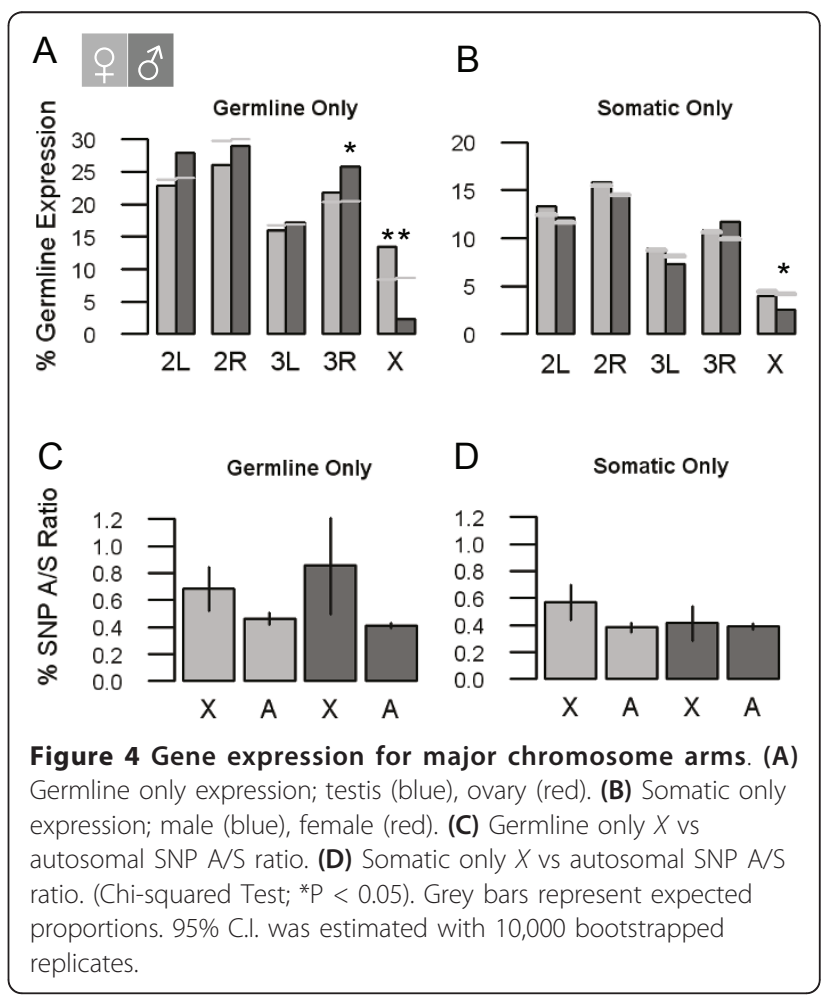




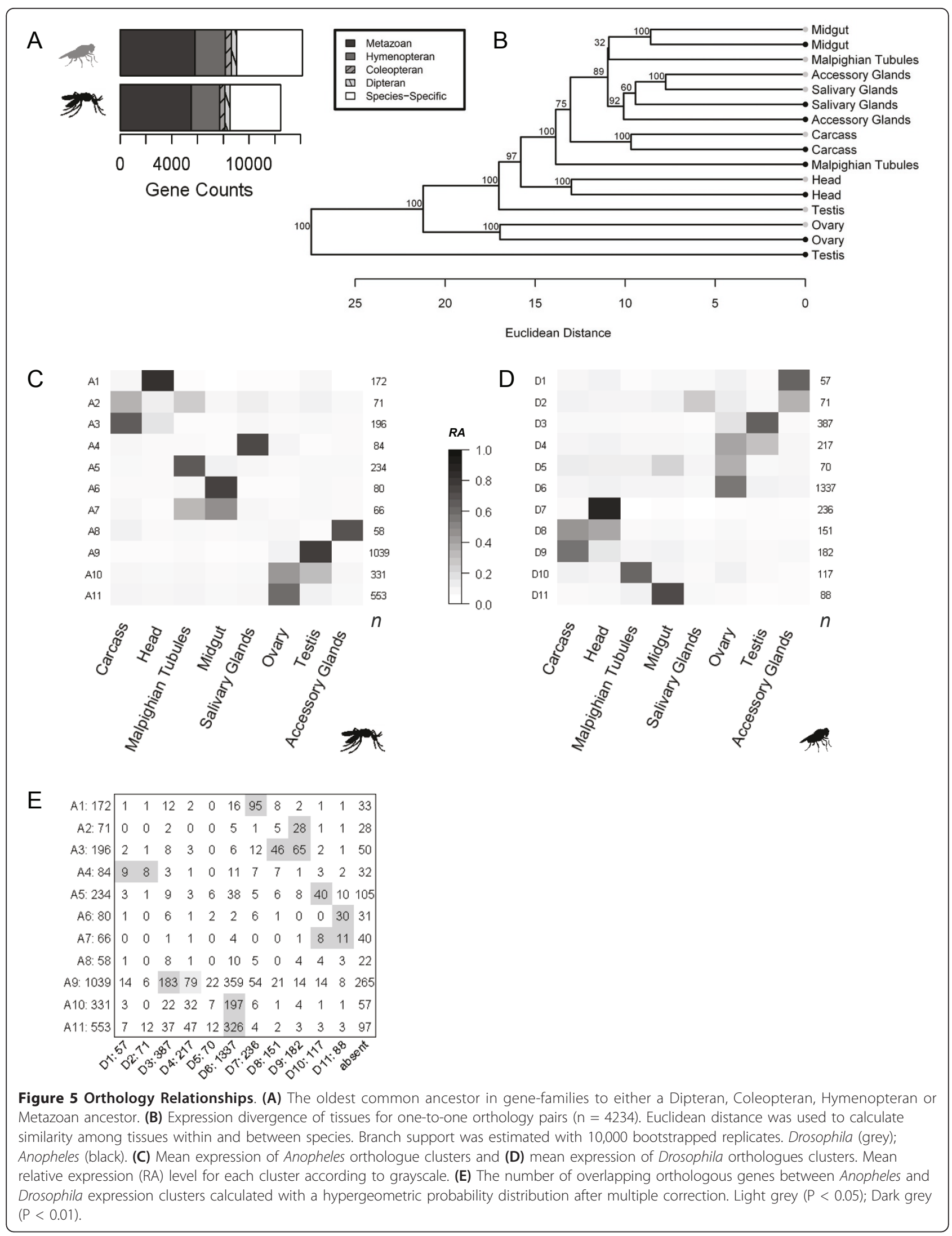


in the Anopheles and Drosophila genomes are in 1:1 orthology relationships ( $\mathrm{n}=6726$; Additional File 5); 95\% of which can be traced back to one of the outgroups used in our analysis with the remaining pairs specific to the Dipteran clade. Using these orthologues, we first compared mosquito expression with the same tissues in Drosophila [19]. Rather than relying on an absolute measure of gene expression, relative measures of abundance (RA) were calculated for each gene (See Methods). Hierarchical clustering of RA across gene pairs showed that global patterns of expression in homologous organs were often more similar between species than between unrelated tissues within a species (Figure 5B). For some organs (i.e. ovary, gut carcass, head), a large proportion of transcriptional variation was conserved between Anopheles and Drosophila, suggesting that the underlying gene networks have similar functional constraints.

To identify conserved expression signatures underling the above patterns, we used hierarchical clustering with pair-wise correlation coefficients to identify co-expressed genes for each species. We chose clusters with an average similarity of greater than 0.8 and more than 50 genes for further analysis. Overall, 11 clusters meet these criteria in Anopheles and Drosophila, representing 2884 and 2913 genes respectively (Figure 5C-D; Additional File 6). Adjusting these thresholds, changes the number of groups identified, but were selected to provide a dataset with reasonably sized gene clusters of highly similar expression profiles.

Between species, we evaluated orthologues in each cluster and found several groups with significant overlap (Figure 5E). Typically, co-expression groups are elevated in one or two tissues. For example, a significant number of orthologues are expressed in the head of both the Anopheles A1 cluster and the Drosophila D7 cluster (Figure 5E). Gene Ontology (GO) annotations for these genes are enriched for 'phototransduction' and 'signal transduction', indicating a close associated with normal physiological functions within head (Table 1). We also found conserved signatures that correspond to expression in the Malpighian tubules, midgut and carcass. A notable exception is that Anopheles salivary gland expression (A4), shares most enrichment with Drosophila orthologues from the male accessory gland (D1, D2). Overall, the largest clusters are expressed in reproductive tissues (Figure 5C-D). Orthologues with testis expression in Anopheles, are spread over a number of Drosophila clusters. We further note that a large proportion of orthologues are expressed in the female ovary. Typically, clusters with elevated ovary expression show significant overlap between Anopheles and Drosophila, and as expected, over-represented GO annotations involve basic cellular processes (Table 1 ).
Table 1 Orthology cluster overlap, tissue expression and enriched gene ontology annotations

\begin{tabular}{|c|c|c|c|}
\hline Anopheles & Drosophila & Tissue & GO \\
\hline A1 & D7 & Head & $\begin{array}{l}\text { phototransduction } \\
\text { signal transduction }\end{array}$ \\
\hline $\begin{array}{l}\mathrm{A} 2 \\
\mathrm{~A} 3\end{array}$ & $\begin{array}{l}\text { D9 } \\
\text { D8, D9 }\end{array}$ & Carcass & $\begin{array}{l}\text { metabolic process } \\
\text { cellular respiration }\end{array}$ \\
\hline A4 & D1, D2 & $\mathrm{SG}, \mathrm{AG}$ & $\begin{array}{l}\text { protein folding } \\
\text { signal peptide processing }\end{array}$ \\
\hline $\begin{array}{l}\text { A5 } \\
\text { A6 } \\
\text { A7 }\end{array}$ & $\begin{array}{l}\text { D10 } \\
\text { D11 } \\
\text { D10, D11 }\end{array}$ & $\begin{array}{l}\text { MT } \\
\text { Midgut } \\
\text { Midgut/MT }\end{array}$ & $\begin{array}{l}\text { transmembrane transport } \\
\text { carbohydrate metabolic process }\end{array}$ \\
\hline A9 & D3, D4 & Testis & $\begin{array}{l}\text { microtubule-based process } \\
\text { spermatogenesis }\end{array}$ \\
\hline $\mathrm{A} 10, \mathrm{~A} 11$ & D6 & Ovary & $\begin{array}{l}\text { nucleic acid metabolic process } \\
\text { oogenesis, cell cycle } \\
\text { eggshell formation }\end{array}$ \\
\hline
\end{tabular}

SG = salivary gland; $\mathrm{AG}=$ Accessory Gland; $\mathrm{MT}=$ Malpighian tubules . Gene ontology significance level $(P<0.01)$.

\section{Single-copy and multi-copy gene families}

Our phylogenetic analysis indicated that 5932 families contain a single Anopheles gene, whereas another 971 families show evidence of Anopheles expansion. In this latter set, duplications with narrow expression patterns (i.e. tau-statistic $>0.85$ ), often arose during the Dipteran split and are most prevalent in the male testis (Figure 6A). However, as well as the testis, a high incidence of duplication events are genes with salivary gland, midgut or Malpighian tubule restricted expression (Figure 6A). Within single-copy families, 143 groups are narrowly expressed in the same Anopheles and Drosophila tissues (Figure 6B). Such expression is prevalent with head and testis expression, but while these genes might be expected to evolve rapidly, the majority date back to Metazoan and Hymenopteran clades.

\section{Online MozAtlas Database}

For researchers interested in comparing their own experiments to the MozAtlas, we have constructed an online database and web-browser for querying tissue expression in Anopheles (http://www.tissue-atlas.org). The single gene query displays tables of normalized expression for each probe and tissue available. In addition, this search displays available orthology relations, a) one-to-one Drosophila melanogaster orthologues and corresponding relative gene expression estimates, and b) a gene tree of all mosquito, fly and outgroups within the gene family. We also provide a BLAST and batch searching facilities to output expression values for larger lists of genes that may then be used for further downstream analysis. 

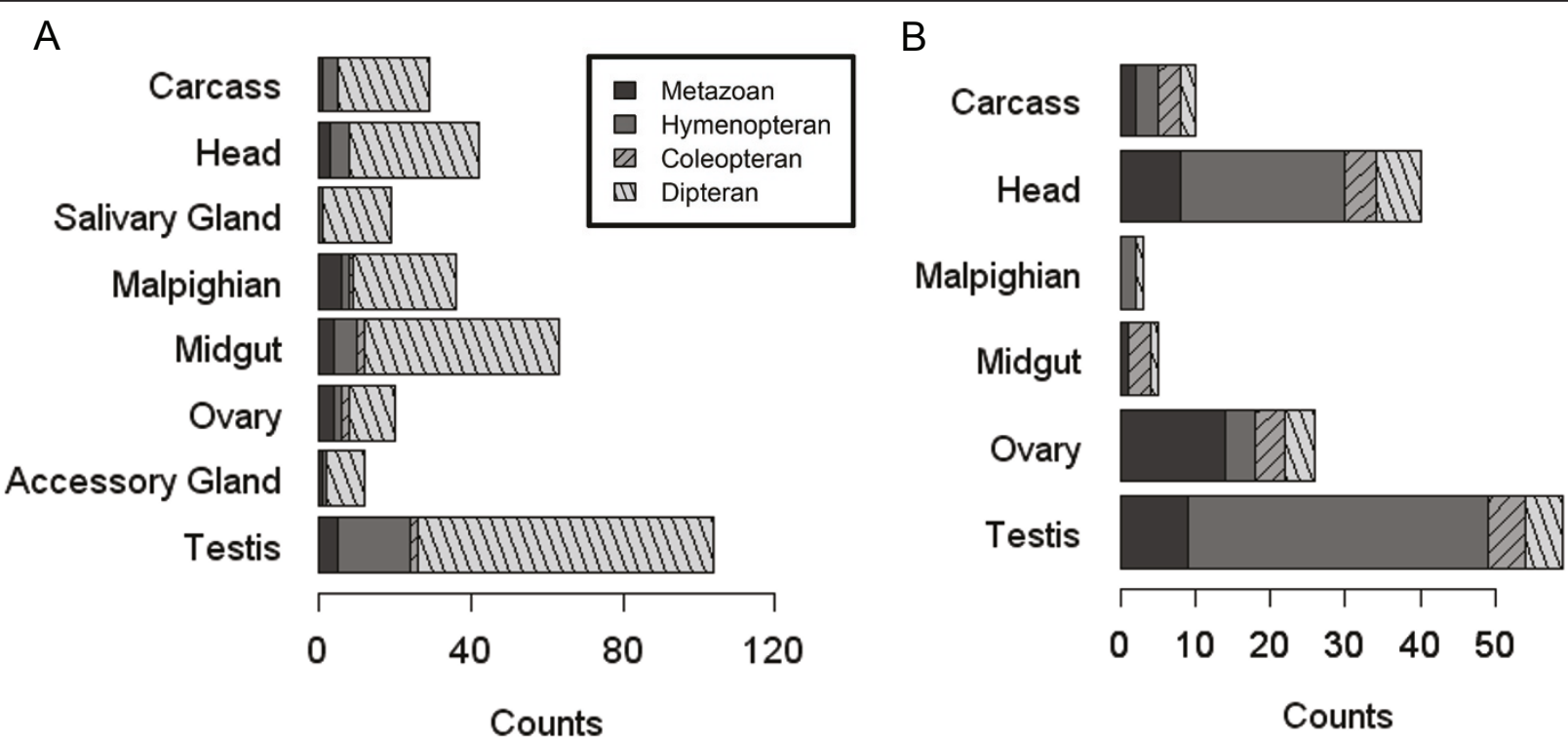

Figure 6 Gene copies, family origins and tissue expression. (A)Anopheles gene expansions with restricted expression patterns ( $\mathrm{n}=325$; taustatistic $=1$ ). (B) Single-copy gene-families with narrow spatial expression profiles in both Drosophila and Anopheles tissues ( $\mathrm{n}=143$; tau-statistic $>0.85)$.

\section{Discussion}

To help improve the functional annotation of the Anopheles gambiae genome we have generated the MozAtlas, a unified catalogue of tissue-specific gene expression from a single mosquito strain. In Drosophila melanogaster, cataloguing tissue expression patterns has been useful, especially for inferring biological functions, since the majority of genes encoded in the genome are not ubiquitously expressed [19]. As with the fruit fly, Anopheles gene expression also exhibits substantial tissue specificity, with only a third of detectably expressed genes found in all tissues. Thus, the MozAtlas is a useful resource for better understanding the mosquito genome, providing direct evidence of genes with tissue restricted expression. Below we highlight the utility of MozAtlas for identifying classes of gene with tissue or sex-biased expression that may be exploited for vector control. Analysis of the MozAtlas also identifies gene expression features that are of interest from an evolutionary perspective, revealing both highly conserved and speciesspecific aspects of insect biology. Of particular interest, given that malaria parasites are only transmitted through female mosquitoes, we separately catalogued gene expression for each tissue in males and females, thus providing both tissue and sex-specific views of gene expression in the adult.

A major finding from our analysis is the substantial degree of sexually dimorphic gene expression we find at the tissue level: more than half of the genes for which we detect expression exhibit sexual dimorphism in terms of expression level. The head, in particular, has a significantly higher number of female-biased genes and of these, odorant receptors are significantly over-represented (Additional File 4). When searching for a bloodmeal, female mosquitoes are attracted to odours emitted by humans, a behaviour mediated by receptors in the antennal sensilla [7]. This activity is not exhibited by males, who feed entirely on nectar, and we presume that the female elevated expression of odorant binding molecules reflect this biology. The identification of molecules associated with female-specific aspects of odorant detection may provide targets for controlling malaria transmission [20].

We identified other sexually dimorphic expression signatures that appear to be associated with female characteristics, in particular, adaptation to hematophagy. For example in the female salivary gland we found an overrepresentation of genes with protein and lipid catabolic activity, ion transport and cellular homostasis functions. We suggest that these reflect the fact that, in females, the salivary gland produces compounds to disarm host hemostatic and immune responses, thus allowing mosquitoes to take a blood-meal. Similarly, many proteins found in the midgut are only synthesized by blood-feeding females [3,21]: numerous digestive and proteolytic molecules implicated in blood digestion were identified as female elevated in our analysis.

In contrast, elevated male gene activity is largely associated with carbohydrate metabolism and ion transport activity. Since male mosquitoes feed entirely on sugar, 
these results were not surprising. However, somewhat more novel is that iron binding molecules are up-regulated in males. While in female mosquitoes iron is especially important for egg development and is strongly influenced by blood-feeding [22], iron metabolism has diverse physiological and developmental roles [23]. Although females obtain iron from the blood meal, the sugar diet of males may necessitate more efficient iron uptake and up-regulation of genes that encode iron binding functions.

In both somatic and reproductive tissues, we identified genes with considerable specificity. Tightly controlled, tissue-specific expression is of interest for understanding the basic biology of a species, and is likely to be key in the development of next generation insect control agents. For example, genes uniquely expressed in particular tissues could be targets for inducing sterility or providing regulatory elements to drive localised expression of transgenes. In this respect, the highest proportion of Anopheles tissue-specific expression is in the testis, with approximately $10 \%$ of transcription uniquely detected in this tissue. Testis specific expression of genes with important roles in spermatogenesis, sperm competition or sperm-egg interactions present a set of targets with potential for inducing male sterility.

After mating, Anopheles females undergo distinct behavioural and physiological changes due to the transfer of both sperm and proteins produced in the male accessory glands [24]: proteins secreted by males and passed to females in seminal fluid could provide a route for altering female fertility. Via specific expression profiling of accessory glands we have identified a new set of potential Anopheles Acp genes that will enable further investigation of sexual conflict within the mosquito. Sexual antagonism between males and females may be expected to cause rapid Acp sequence evolution [25]. We find that among tissue-specific genes, those expressed in the accessory gland have a higher $\mathrm{A} / \mathrm{S}$ ratio than in many tissues, including the testis. Slower evolutionary rates in the Anopheles testis might be explained, in part, by their mating behaviour: in polyandrous insects genes involved in spermatogenesis are often under strong positive selection as a result of post-copulatory male-male competition [25], whereas these pressures in the testis are expected to be absent from the largely monandrous Anopheles mosquitoes [26].

Genes with ovary specific expression provide potential targets for inducing female sterility in mosquitoes given that they are closely associated with egg formation. Chorion components of the fruit fly eggshell, for example, provide the embryo with protection from the physical environment, and disrupting their function causes female sterility [27]. Recently, proteomic techniques have identified Anopheles eggshell constituents, several of which we find to be specifically expressed in the ovary, making them favourable candidates for use in population control [28].

In terms of genome structure, we show that genes with male-biased expression are non-randomly distributed around the Anopheles genome. Two mechanisms have been proposed to explain the disparity in chromosomal distribution of male expressed genes. First, during spermatogenesis the $X$ chromosome of males becomes inactivated: since few testis genes are expressed postmeiotically, evidence suggests that chromosomal inactivation has promoted autosomal duplication events from $X$-linked genes $[18,29,30]$. There is compelling evidence that $X$-linked inactivation also occurs in nematodes [31] and mammals [32], however, an under-representation of male-biased somatically-expressed genes on the $X$ chromosome indicates that other forces are also at work. Second, since males only have one $X$ chromosome, polymorphisms beneficial to one sex may arise that are detrimental to the other sex. Such antagonistic sexual selection may eventually lead to sequence changes and demasculinization of the $X$ chromosome [33], and consistent with this expectation, genes on the Anopheles $X$ chromosome have less sequence polymorphism than on the autosomes.

Identifying expression divergence within and between closely-related species provides important insights into the selective pressures underlying gene regulation $[34,35]$. The opportunity to compare divergence between Drosophila and Anopheles, separated by some 250 million years of evolution, allows us to explore gene and tissue evolution over a considerable time scale. We find that expression similarity in one-to-one orthologues of the midgut, head, carcass and ovary expressed genes is well conserved in the Diptera and, as expected, genes in conserved co-expression clusters perform integral physiological functions.

In contrast, tissues such as the testis, often show considerable transcriptional variation between closely related species [36,37]. It's been proposed that testis gene regulation plays a critical role in the initial formation of reproductive isolation [38]. In addition to the Anopheles testis, expression in other tissues is also highly divergent: for example, expression in the Malpighian tubules is largely not conserved between Anopheles and Drosophila. As an organ with a key role in detoxification and osmoregulation, this divergence may reflect fundamental differences in the diet of each insect [39]. In addition, salivary gland and male accessory gland expression cluster within rather than between species, evidence for a bout of simultaneous evolution since the last common ancestor was shared. Indeed, no 
significant co-expression was detected between species, indicating that secretory organ functions have diverged during the Dipteran split.

Recent Anopheles gene duplications are often expressed in the testis and, in Drosophila, extreme expansions also have spermatogenesis-related functions [40]. As well as the testis, other tissues display narrow expression profiles of recent origin in Anopheles. Certainly, the blood meal imposes a range of challenges on the digestive system of mosquitoes and, in part, explains a predominance of gene duplications with salivary gland, Malpighian tubule or midgut expression. Even between members of the same mosquito subgenera, salivary proteins can diverge rapidly over time [41]: our data suggests that this evolutionary pattern may also be common in Malpighian tubule proteins and, to a lesser extent, proteins within the midgut. However, specifically expressed genes in large families do not necessarily highlight unique functions, since homologues may perform the same or similar functions in a larger set of tissues. Gene families with single members are of interest for identifying unique processes, given that closely related homologues are not found within the genome. Narrowly expressed singlecopy families were detected dating back to Metazoan and Hymenopteran clades, perhaps accompanying the emergence of differentiated organs. It will be of considerable interest for insect control programs to determine whether such proteins perform integral functions in their specific tissues, given that as single copies they should perform unique roles within the organism.

\section{Conclusions}

We have generated a tissue and sex-specific gene expression atlas for Anopheles gambiae and used it to explore mosquito biology related to reproduction, feeding and gene evolution. Given that Anopheles is the major vector of one of the world's most debilitating diseases, our dataset provides an important reference for other mosquito researchers wishing to explore potential roles for genes of interest. Of particular importance is the identification of uniquely expressed genes that may serve as tissue-specific drivers in transgenic constructs or potential knockout targets in the next generation of insect control agents.

\section{Methods}

\section{RNA collections and microarray platform}

Male and female mosquito siblings were separated at pupation and allowed to emerge into separate cages to prevent mating. 3-day old, non-mated females were blood-fed and female tissues were dissected at 24 hour intervals for a three day period following the bloodmeal. Equivalent male tissues were dissected from agematched siblings in parallel. Dissections were carried out in phosphate-buffered saline using dissecting needles and a 28 gauge needle to cleanly separate connected tissues from each other. 'Midgut' samples were dissected clear of the foregut, hindgut and malphigian tubules to include the anterior midgut and stomach. 'Head' samples were produced by severing at the neck and include brain, eyes, cuticle and some fat body. 'Ovary' samples include both ovaries and the common oviduct. 'Salivary gland' samples include the salivary duct, lateral lobes and median lobe. Salivary glands were rinsed extensively in PBS to remove the majority of fat body associated with the glands. 'Carcass' includes the thoracic and abdominal carcass and all tissues therein excluding those tissues individually described in the MozAtlas. Dissected tissues were placed immediately in Trizol to minimize the impact of dissection on the transcriptome. For each of four biological replicates, tissues were pooled from a minimum of 10 mosquitoes dissected at each time point. For each tissue and sex, an equal quantity of total RNA was pooled from three time points sampled after the blood-meal to obtain gene expression estimates throughout oogenesis (24, 48, 72 hrs). Each RNA sample (50 ng) was subsequently amplified in two cycle cDNA target labelling to generate biotinylated cRNA probes for hybridization on to Affymetrix microarrays [42].

\section{Estimates of gene expression}

Oligonucleotide probes and genes were mapped to AgamP3 genome assembly. Unless otherwise stated, datasets were analyzed with the $\mathrm{R}$ statistical programming language using programs maintained as part of the Bioconductor suite [43]. In addition to microarray datasets for Anopheles, matching tissues obtained from the Drosophila FlyAtlas were re-analyzed with the same normalization procedure (GEO: GSE1690; GSE7763). Intensity values between arrays were first standardized within tissues for each species separately using the robust multi-array analysis package $[44,45]$. The expression presence and absence calls were assessed with the signal to noise ratio of the perfect match and mismatch probes provided on Affymetrix arrays. Probes were used in further analysis only if they were deemed to be present in at least three tissue replicates. All estimates of differential expression were adjusted for multiple testing using the false discovery rate method [46]. Array data has been submitted to the Gene Expression Omnibus under GSE21689.

\section{Sexual dimorphism and tissue specificity}

Sexual dimorphism was determined with a linear model of gene expression fit to male and female samples for each tissue as implemented in the LIMMA library [47]. On the basis of differential expression, we subsequently 
identified probes as either male-biased or female-biased where there was a significant 2-fold change of intensity in one sex, in addition to statistical significance at the $\mathrm{Q}<0.05$ level (Additional File 3). Two measures of tissue specificity were also calculated: probe detection and tissue expression breadth. Probes were deemed tissuespecific if at least 3 out of 4 mismatch calls were found, but only in a single tissue and sex. In comparison, tissue breadth was measured by normalizing against maximal expression to generate the tau-statistic [48]. The resulting tau-statistic falls within the range of 0 to 1 , in which higher values indicate greater tissue-biased expression. Anopheles and Drosophila Gene Ontology annotations (Biological Process, Molecular Function, Cellular Component) and the enrichment of functions were determined using FlyMine with a $1 \%$ false discovery rate for multiple testing correction [49].

\section{SNP polymorphism}

When sequences are available for multiple individual in a species, the ratio of observed non-synonymous mutation rate (A) to the synonymous mutation rate (S) can be utilized as an estimate of the selective pressure. To estimate sequence polymorphism within Anopheles we conducted a large-scale survey of dbSNP [17]. While it is not possible to measure selective constraint on individual proteins directly using this approach, it has been demonstrated that when a group of genes are measured together, estimates of variation are robust and in good agreement with $\mathrm{A} / \mathrm{S}$ for divergence [50].

\section{Expression divergence}

Since microarray platforms were designed separately for Drosophila and Anopheles, probes have different affinities to their target RNAs, making the normalization of orthology expression between chips difficult. In order to compare tissue expression profiles between species, each gene was represented as a vector of relative expression abundance (RA) across the sampled tissues to avoid over-estimating divergence based on absolute expression intensity. Where genes are represented by multiple probes, the maximum intensity value recorded in each tissue was used for subsequent analysis. Since the FlyAtlas does not have separate samples for males and females, we combined male and female samples in the MozAtlas to make comparisons between species. Hierarchical clustering of orthologues was performed with measures of RA within and between species. For genewise clustering, we used Pearson correlation coefficient as the distance measure and defined similarity between clusters using average-linkage clustering. Co-regulated genes were defined as any group with an average similarity of greater than 0.8 that also contained more than 50 genes. Among species clusters, orthologue overlap was subsequently investigated with a hypergeometric probability distribution to determine enrichment.

\section{Orthology classification}

DNA and protein sequences were obtained for D. melanogaster and A. gambiae (Ensembl v50) [51], Tribolium casteneum (Version 3; BeetleBase) [52], Apis melifera (Version 2; BeeBase) [53] and Caenorhabditis elegans (ws160; Ensembl v50) [51]. One-to-one orthology relationships were determined using Inparanoid with default parameters, we selected the longest available translation for each annotated protein [54]. Best reciprocal hits between species were grouped together into broader gene-families, and the sequences aligned with MUSCLE [55]. Tree topologies were subsequently reconstructed with both dS (synonymous substitution rate), $\mathrm{dN}$ (nonsynonymous substitution rate), nucleotide and protein distance measures using TreeBest [56,57]. From back-translation of protein alignments, TreeBest creates a consensus tree by merging the results of neighbour joining and maximum likelihood $(\mathrm{ML})$ trees. By default, ML trees based on protein alignment are built under the WAG model, while ML tree based on DNA are built under the HKY model, which models non-uniform base composition and transition/transversion rate bias [58]. Orthology relationships are described as one-to-one, one-to-many and many-to-many gene relationships.

\section{Acknowledgements}

This study was funded by a Grant from the Foundation for the National Institutes of Health through the Grand Challenges in Global Health initiative.

\section{Additional material}

Additional file 1: Figure S1 - Correlation of gene expression
between replicate samples.
Additional file 2: Figure S2 - Hierarchical clustering of probe
intensity.
Additional file 3: Figure S3 - Sexual dimorphism of tissue gene
expression.
Additional file 4: Table S1 - Gene Ontology annotation of sexual
dimorphic gene expression.
Additional file 5: Table S2 - One-to-one orthology relationships and
gene expression.
Additional file 6: Table S3 - Cluster annotation of one-to-one
orthology relationships.

\section{Author details}

'Department of Genetics, University of Cambridge, Downing Street, Cambridge CB1 3QA, UK. 'Department of Life Sciences, Sir Alexander Fleming Building, Imperial College London, Imperial College Road, London SW7 2AZ, UK. ${ }^{3}$ Cambridge Systems Biology Centre, Tennis Court Road, Cambridge CB2 1QR, UK. 


\section{Authors' contributions}

DAB conceived, analysed and designed the study, performed microarray experiments with $\mathrm{BF}$, and wrote the paper. TN and AP reared the mosquitoes and dissected the tissue samples. TN, SR and AC participated in the design of this study and helped write the paper. AC and SR raised the funding. All authors read and approved the final document.

Received: 31 January 2011 Accepted: 7 June 2011

Published: 7 June 2011

\section{References}

1. Hahn MW, Lanzaro GC: Female-biased gene expression in the malaria mosquito Anopheles gambiae. Curr Biol 2005, 15:R192-193.

2. Marinotti $\mathrm{O}$, et al: Genome-wide analysis of gene expression in adult Anopheles gambiae. Insect Mol Biol 2006, 15:1-12.

3. Dana AN, Hong YS, Kern MK, Hillenmeyer ME, Harker BW, Lobo NF, Hogan JR, Romans P, Collins FH: Gene expression patterns associated with blood-feeding in the malaria mosquito Anopheles gambiae. BMC Genomics 2005, 6:5.

4. Arcà B, Lombardo F, Valenzuela JG, Francischetti IM, Marinotti O, Coluzzi M, Ribeiro JM: An updated catalogue of salivary gland transcripts in the adult female mosquito, Anopheles gambiae. J Exp Biol 2005, 208:3971-86.

5. Calvo E, Pham VM, Lombardo F, Arcà B, Ribeiro JM: The sialotranscriptome of adult male Anopheles gambiae mosquitoes. Insect Biochem Mol Biol 2006, 36:570-5

6. Koutsos AC, Blass C, Meister S, Schmidt S, MacCallum RM, Soares MB, Collins FH, Benes V, Zdobnov E, Kafatos FC, Christophides GK: Life cycle transcriptome of the malaria mosquito Anopheles gambiae and comparison with the fruitfly Drosophila melanogaster. Proc Natl Acad Sci USA 2007, 104:11304-9.

7. Biessmann H, Nguyen QK, Le D, Walter MF: Microarray-based survey of a subset of putative olfactory genes in the mosquito Anopheles gambiae. Insect Mol Biol 2005, 14:575-89.

8. latrou K, Biessmann $\mathrm{H}$ : Sex-biased expression of odorant receptors in antennae and palps of the African malaria vector Anopheles gambiae. Insect Biochem Mol Biol 2008, 38:268-74.

9. Burt A: Site-specific selfish genes as tools for the control and genetic engineering of natural populations. Proc Biol Sci 2003, 270:921-928.

10. Heinrich J, Scott M: A repressible female-specific lethal genetic system for making transgenic insect strains suitable for a sterile-release program. Proc Natl Acad Sci USA 2000, 97:8229-8232.

11. Thomas DD, Donnelly CA, Wood RJ, Alphey LS: Insect population control using a dominant, repressible, lethal genetic system. Science 2000, 287:2474-2476.

12. Franz AW, Sanchez-Vargas I, Adelman ZN, Blair CD, Beaty BJ, James AA, Olson KE: Engineering RNA interference-based resistance to dengue virus type 2 in genetically modified Aedes aegypti. Proc Natl Acad Sci USA 2006, 103:4198-4203.

13. Phuc HK, Andreasen MH, Burton RS, Vass C, Epton MJ, Pape G, Fu G, Condon KC, Scaife S, Donnelly CA, et al: Late-acting dominant lethal genetic systems and mosquito control. BMC Biol 2007, 5:11.

14. Windbichler N, Papathanos PA, Catteruccia F, Ranson H, Burt A, Crisanti A: Homing endonuclease mediated gene targeting in Anopheles gambiae cells and embryos. Nucleic Acids Res 2007, 35:5922-5933.

15. Fu G, Lees RS, Nimmo D, Aw D, Jin L, Gray P, Berendonk TU, WhiteCooper H, Scaife S, Kim Phuc H, et al: Female-specific flightless phenotype for mosquito control. Proc Natl Acad Sci USA 2010, 107:4550-4.

16. Larracuente AM, Sackton TB, Greenberg AJ, Wong A, Singh ND, Sturgill D, Zhang $Y$, Oliver B, Clark AG: Evolution of protein-coding genes in Drosophila. Trends Genet 2008, 24:114-123.

17. Sherry ST, et al: dbSNP: the NCBI database of genetic variation. Nucleic Acids Res 2001, 29:308-311.

18. Sturgill $D$, Zhang $Y$, Parisi M, Oliver B: Demasculinization of $x$ chromosomes in the Drosophila genus. Nature 2007, 450:238-241.

19. Chintapalli VR, Wang J, Dow JA: Using FlyAtlas to identify better Drosophila melanogaster models of human disease. Nat Genet 2007, 39:715-720.

20. Carey AF, Wang G, Su C, Zwiebel L, Carlson JR: Odorant reception in the malaria mosquito Anopheles gambiae. Nature 2010, 464:66-71.
21. Warr E, Aguilar R, Dong Y, Mahairaki V, Dimopoulos G: Spatial and sexspecific dissection of the Anopheles gambiae midgut transcriptome. BMC Genomics 2007, 8:37.

22. Dunkov B, Georgieva T: Insect iron binding proteins: insights from the genomes. Insect Biochem Mol Biol 2006, 36:300-309.

23. Nichol H, Law JH, Winzerling JJ: Iron metabolism in insects. Annu Rev Entomol 2002, 47:535-559.

24. Rogers DW, Whitten MM, Thailayil J, Soichot J, Levashina EA, Catteruccia F: Molecular and cellular components of the mating machinery in Anopheles gambiae females. Proc Natl Acad Sci USA 2008, 105:19390-19395.

25. Haerty W, Jagadeeshan S, Kulathinal RJ, Wong A, Ravi Ram K, Sirot LK, Levesque L, Artieri CG, Wolfner MF, Civetta A, et al: Evolution in the fast lane: rapidly evolving sex-related genes in Drosophila. Genetics 2007, 177:1321-1335

26. Tripet $F$, Touré $Y T$, Dolo G, Lanzaro GC: Frequency of multiple inseminations in field-collected Anopheles gambiae females revealed by DNA analysis of transferred sperm. Am J Trop Med Hyg 2003, 68:1-5.

27. Galanopoulos VK, Orr W, Szabad J, Kafatos FC: Genetic analysis of chorion formation in Drosophila melanogaster: I. The effects of one somaticspecific and seven germ-line-specific mutations. Dev Genet 1989, 10:87-97.

28. Amenya DA, Chou W, Li J, Yan G, Gershon PD, James AA, Marinotti O: Proteomics reveals novel components of the Anopheles gambiae eggshell. J Insect Physiol 2010, 56:1414-1419.

29. Vibranovski MD, Zhang Y, Long M: General gene movement off the $\times$ chromosome in the Drosophila genus. Genome Res 2009, 19:897-903.

30. Kemkemer $C$, Hense $W$, Parsch J: Fine-scale analysis of $\times$ chromosome inactivation in the male germline of Drosophila melanogaster. Mol Biol Evol 2010

31. Bean CJ, Schaner CE, Kelly WG: Meiotic pairing and imprinted $x$ chromatin assembly in Caenorhabditis elegans. Nat Genet 2004, 36:100-105.

32. Khil PP, Smirnova NA, Romanienko PJ, Camerini-Otero RD: The mouse $x$ chromosome is enriched for sex-biased genes not subject to selection by meiotic sex chromosome inactivation. Nat Genet 2004, 36:642-646.

33. Wu Cl, Xu EY: Sexual antagonism and $\times$ inactivation-the SAXI hypothesis. Trends Genet 2003, 19:243-247.

34. Khaitovich P, Hellmann I, Enard W, Nowick K, Leinweber M, Franz H, Weiss $G$, Lachmann $M, P$ ääbo $S$ : Parallel patterns of evolution in the genomes and transcriptomes of humans and chimpanzees. Science 2005, 309:1850-1854.

35. Zhang Y, Sturgill D, Parisi M, Kumar S, Oliver B: Constraint and turnover in sex-biased gene expression in the genus Drosophila. Nature 2007 450:233-237.

36. Ranz JM, Castillo-Davis Cl, Meiklejohn CD, Hartl DL, et al: 2003, Sexdependent gene expression and evolution of the Drosophila transcriptome. Science 2003, 300:1742-1755.

37. Meiklejohn CD, Parsch J, Ranz JM, Hartl DL: Rapid evolution of malebiased gene expression in Drosophila. Proc Natl Acad Sci USA 2003, 100:9894-9899

38. Voolstra C, Tautz D, Farbrother P, Eichinger L, Harr B: Contrasting evolution of expression differences in the testis between species and subspecies of the house mouse. Genome Res 2007, 17:42-49.

39. Wang J, Kean L, Yang J, Allan AK, Davies SA, Herzyk P, Dow JA: Functioninformed transcriptome analysis of Drosophila renal tubule. Genome Biol 2004, 5:R69.

40. Hahn MW, Han MV, Han SG: Gene family evolution across 12 Drosophila genomes. PLoS Genet 2007, 3:e197.

41. Arcà B, Lombardo F, Francischetti IM, Pham VM, Mestres-Simon M, Andersen JF, Ribeiro JM: An insight into the sialome of the adult female mosquito Aedes albopictus. Insect Biochem Mol Biol 2007, 37:107-27.

42. Affymetrix GeneChip Expression Analysis: Technical Manual. 701021 Rev. 5. Santa Clara, CA, Affymetrix; 2004.

43. Gentleman RC, Carey VJ, Bates DM, Bolstad B, Dettling M, Dudoit S, Ellis B, Gautier L, Ge Y, Gentry J, Hornik K, et al: Bioconductor: open software development for computational biology and bioinformatics. Genome Biol 2004, 5:R80.

44. Irizarry RA, et al: Exploration, normalization, and summaries of high density oligonucleotide array probe level data. Biostatistics 2003 4:249-264 
45. Wu Z, Irizarry RA, Gentleman R, Martinez-Murillo F, Spencer F: A ModelBased Background Adjustment for Oligonucleotide Expression Arrays. $J$ Am Stat Assoc 2004, 99:909.

46. Hochberg $Y$ : Controlling the false discovery rate: a practical and powerful approach to multiple testing. J Royal Stat Soc B 1995, 57:289-300.

47. Smyth GK: Linear models and empirical bayes methods for assessing differential expression in microarray experiments. Stat Appl Genet Mol Biol 2004, 3:Article 3

48. Yanai I, Benjamin H, Shmoish M, Chalifa-Caspi V, Shklar M, Ophir R, BarEven A, Horn-Saban S, Safran M, Domany E, et al: Genome-wide midrange transcription profiles reveal expression level relationships in human tissue specification. Bioinformatics 2005, 21:650-659.

49. Lyne R, Smith R, Rutherford K, Wakeling M, Varley A, Guillier F, Janssens H, Ji W, Mclaren P, North P, et al: FlyMine: an integrated database for Drosophila and Anopheles genomics. Genome Biol 2007, 8:R129.

50. Liu J, Zhang $Y$, Lei $X$, Zhang Z: Natural selection of protein structural and functional properties: a single nucleotide polymorphism perspective. Genome Biol 2008, 9:R69.

51. Flicek P, Aken BL, Ballester B, Beal K, Bragin E, Brent S, Chen Y, Clapham P, Coates G, Fairley S, et al: Ensembl's 10th year. Nucleic Acids Res 2010, 38 : D557-562.

52. Wang L, Wang S, Li Y, Paradesi MS, Brown SJ: BeetleBase: the model organism database for Tribolium castaneum. Nucleic Acids Res 2007, 35 D476-479.

53. Elsik CG, Worley KC, Zhang L, Milshina NV, Jiang H, Reese JT, Childs KL, Venkatraman A, Dickens CM, Weinstock GM, et al: Community annotation: procedures, protocols, and supporting tools. Genome Res 2006, 16:1329-1333.

54. O'Brien KP, Remm M, Sonnhammer EL, Inparanoid : A Comprehensive Database of Eukaryotic Orthologs. NAR 2005, 33:D476-D480.

55. Edgar RC: MUSCLE: a multiple sequence alignment method with reduced time and space complexity. BMC Bioinformatics 2004, 5:113.

56. TreeSoft: Softwares for Phylogenetic Trees. [http://treesoft.sourceforge. net/treebest.shtml].

57. Ruan J, et al: TreeFam: Update. Nucleic Acids Res 2008, 36:D735-D740.

58. Guindon S, Lethiec F, Duroux P, Gascuel O: PHYML Online-a web server for fast maximum likelihood-based phylogenetic inference. Nucleic Acids Res 2005, 33:W557-W559.

doi:10.1186/1471-2164-12-296

Cite this article as: Baker et al:: A comprehensive gene expression atlas of sex- and tissue-specificity in the malaria vector, Anopheles gambiae. BMC Genomics 2011 12:296.

\section{Submit your next manuscript to BioMed Central and take full advantage of:}

- Convenient online submission

- Thorough peer review

- No space constraints or color figure charges

- Immediate publication on acceptance

- Inclusion in PubMed, CAS, Scopus and Google Scholar

- Research which is freely available for redistribution 